\title{
Modified coupled-mode model for thermally chirped polymer Bragg gratings
}

\author{
Ilai Sher, ${ }^{1, *}$ Bongtae Han, ${ }^{2}$ and Avram Bar-Cohen ${ }^{2}$ \\ 'School of Engineering, Cranfield University, Cranfield, Bedfordshire, MK43 OAL, UK \\ ${ }^{2}$ Department of Mechanical Engineering, University of Maryland at College Park, College Park, Maryland 20742, USA \\ ${ }^{*}$ Corresponding author: i.sher@cranfield.ac.uk
}

Received 30 October 2009; revised 5 March 2010; accepted 6 March 2010; posted 9 March 2010 (Doc. ID 119302); published 5 April 2010

\begin{abstract}
A modified coupled-mode (CM) model is proposed for the optical behavior of thermally chirped Bragg gratings. The model accounts for the axial gradient in the modulation wavenumber, which has been ignored in the classical CM model. The model is used to characterize the optical behavior of a polymethyl methacrylate-based polymer Bragg grating subjected to nonisothermal conditions. The validity of the proposed method is verified by comparing the results of the modified CM model with those obtained from the exact numerical solution. (C) 2010 Optical Society of America

OCIS codes: $\quad 050.1590,060.3735$.
\end{abstract}

\section{Introduction}

Chirped gratings, resulting from natural or intentional variations in the properties and geometries of an otherwise periodic structure, have been extensively treated in the literature [1-14]. Analyses of chirped gratings are usually based on the coupledmode (CM) theory, first proposed by Kogelnik [1].

The direct numerical integration of the $\mathrm{CM}$ equations along chirped gratings, as suggested by Kogelnik [1], has been shown to agree well with experimental results of mildly chirped gratings. Hong et al. [2] have developed asymptotic approximations of the CM equations for linearly chirped gratings. Fukuzawa and Nakamura [3] and Livanos et al. [4] have suggested a chirped grating reflectivity calculation method, which is based on the approximate form of the CM theory. In the approximate form, use is made of an "effective" grating length-defined as the distance over which significant reflectance occurs. Their approximation was found to agree reasonably well with the results of the direct CM numerical integration.

0003-6935/10/112079-06\$15.00/0

(C) 2010 Optical Society of America
Yamada and Sakuda [5] have suggested a method applicable to mildly chirped gratings, or "almost periodic" gratings. Their method is a piecewise-uniform approach, in which the grating is divided into a number of segments of uniform periodicity, the CM equations are solved for each segment, and the results combined in a series of matrices. Erdogan [6] has concluded that the approximation of the piecewiseuniform approach is in good agreement with the solutions of the direct CM numerical integration, for a variety of mildly chirped gratings. A similar approach was taken by Weller-Brophy and Hall [7], who analyzed chirped gratings using the Rouard method, approximating the grating as a stack of a finite number of slabs and determining the effective reflectivity of each slab by the CM theory. At the asymptotic limit, where the grating is represented by an infinite number of slabs, the Rouard approximation becomes exact. Sipe et al. [8] have suggested an "effective properties" description of chirped gratings in the context of the CM equations. A later study by Poladian [9] made use of that "effective properties" description to develop phase integral Wentzel-KramersBrillouin (WKB) approximate solutions of the CM equations for chirped gratings. These have been found to agree with the results of the direct CM numerical integration. 
The thermo-optic effects of intrinsic heating on the performance of polymer fiber gratings have attracted recent attention. Notable examples of such experimental studies include the work of Peng and Chu [10] on the photosensitivities of polymer optical fibers and use of fiber temperature changes to create tunable fiber gratings, the work of Liu et al. [11] on a thermal stability test for polymer fibers, which pointed to a potential compatibility of polymers with such applications, and a more recent study by Littler et al. [12], considering the effect of intrinsic heating on the Bragg shift in an isothermal grating.

Consideration was then extended by Kim et al. [13] to gratings with axial temperature gradients, created by nonuniform intrinsic heating, leading to a nontraditional, "thermally chirped" Bragg grating. When exposed to a temperature gradient, the fiber Bragg grating (FBG) optical properties will be altered in two ways: by a thermo-optic effect (varied, nonuniform, shift in the refractive index unrelated to the Bragg grating) and by a thermomechanical effect (varied, nonuniform, thermal expansion of the material, altering the period of the Bragg grating). The nonuniformity of these effects along the grating causes a chirp in the FBG. A model to precisely characterize the optical behavior of such thermally chirped gratings must consider the axial gradient of the modulation wavenumber, which has been ignored in the original CM model.

In this study, a rigorous approach to modeling of thermal effects on thermally sensitive Bragg gratings is suggested, in which the CM optical equations are rederived for thermally chirped polymer Bragg gratings.

\section{Modeling}

In this analysis the Bragg grating is treated as a large number $(N)$ of sequential elements, with reflection occurring at the interfaces between elements and propagation within the elements. Considering the $j$ th element, the local $j$ th wave amplitudes of the forward $(F)$ and the backward $(R)$ waves are related to the $(j+1)$ th amplitudes through the multiplication of the complex transmission coefficient, the complex reflectivity matrix, and the propagation matrices [14], i.e.,

$$
\left[\begin{array}{l}
F_{j} \\
R_{j}
\end{array}\right]=\frac{1}{\tau_{j}}\left[\begin{array}{cc}
1 & -\rho_{j}^{*} \\
\rho_{j} & 1
\end{array}\right]\left[\begin{array}{cc}
e^{i k_{j} \Delta z} & 0 \\
0 & e^{-i k_{j} \Delta z}
\end{array}\right]\left[\begin{array}{l}
F_{j+1} \\
R_{j+1}
\end{array}\right],
$$

where the complex transmission coefficient and the complex reflectivity coefficient are given, respectively, by

$$
\tau_{j}=\frac{2 n_{j}}{n_{j}+n_{j+1}}, \quad \rho_{j}=\frac{n_{j}-n_{j+1}}{n_{j}+n_{j+1}},
$$

The complex refractive index for any element in the grating can be expressed as the sum of the nonperiodic and the periodic contributions to the refractive index variation, i.e.,

$$
n(z)=n_{0}(z)+\delta n e^{i B(z) z} .
$$

The modulation wavenumber, $B(z)$ varies along the grating, i.e., $B(z)=\frac{2 \pi}{\Lambda(z)}$, where $\Lambda(z)$ is the grating period. The propagation wavenumber may also vary along the grating, according to $k(z)=\frac{2 \pi}{\lambda_{0}} n(z)$, where $\lambda_{0}$ is the wavelength in vacuum.

Subtracting the $(j+1)$ th wave amplitudes vector from both sides of Eq. (1) and allowing the elements to become vanishingly thin, yields a simple differential equation:

$$
d\left[\begin{array}{l}
F \\
R
\end{array}\right]=A\left[\begin{array}{l}
F \\
R
\end{array}\right]
$$

where

$$
A=\left(\begin{array}{cc}
1-\frac{1}{\tau} e^{i k \Delta z} & \frac{\rho^{*}}{\tau} e^{-i k \Delta z} \\
-\frac{\rho}{\tau} e^{i k \Delta z} & 1-\frac{1}{\tau} e^{-i k \Delta z}
\end{array}\right)
$$

The first term of the Taylor series of $\tau, \rho$, and $e^{i k \Delta z}$ can be expressed as

$$
\begin{aligned}
\rho_{j} & =\frac{n_{j}-n_{j+1}}{n_{j}+n_{j+1}} \approx \frac{-n^{\prime} \Delta z}{2 n+n^{\prime} \Delta z}, \\
\tau_{j} & =\frac{2 n_{j}}{n_{j}+n_{j+1}}=\frac{2 n}{2 n+n^{\prime} \Delta z}, \quad e^{i k \Delta z} \approx 1+i k \Delta z
\end{aligned}
$$

where $n^{\prime} \equiv \frac{\mathrm{d} n}{\mathrm{~d} z}$. Substituting these relations into matrix $A$, and taking the first term in $\Delta z$, yields

$$
A=\left(\begin{array}{cc}
-\frac{1}{2} \frac{n^{\prime}}{n}-i k & -\frac{1}{2} \frac{n^{\prime} *}{n} \\
\frac{1}{2} \frac{n^{\prime}}{n} & -\frac{1}{2} \frac{n^{\prime}}{n}+i k
\end{array}\right) \Delta z .
$$

By assuming that the modulated variation of refractive index is small in comparison to its base value (i.e., $\left.\delta n \ll n_{0}(z)\right)$ and that the chirp-induced spatial variation of the base refractive index is much smaller than the modulated spatial variation of the refractive index (i.e., $n_{0}^{\prime}(z) \ll \delta n B(z)$ ), the refractive index terms can be evaluated as

$$
\begin{aligned}
\frac{n^{\prime}(z)}{n(z)} & =\frac{n_{0}^{\prime}(z)+i\left[B(z)+z B^{\prime}(z)\right] \delta n e^{i B(z) z}}{n_{0}(z)+\delta n e^{i B(z) z}} \\
& \approx i \frac{\delta n}{n_{0}(z)}\left[B(z)+z B^{\prime}(z)\right] e^{i B(z) z},
\end{aligned}
$$

where $B^{\prime}(z) \equiv \frac{\mathrm{d} B(z)}{\mathrm{d} z}$. In Eq. (7), explicit notation is used to emphasize $z$-dependent variables. These assumptions can be quantitatively justified for glass and polymer fibers [13] by inserting typical optical properties of glass and polymer (Table 1 [13]).

By substituting Eq. (7) in (6) and making an additional assumption that the numerical value of $\delta n / n_{0}$ is far smaller than $k / B$, i.e., $\frac{\delta n}{n_{0}} \ll \frac{k}{B}$, which can also be quantitatively justified, Eq. (ㅁ) can be expressed as 


$$
A \approx-i\left(\begin{array}{cc}
k(z) & \left.\frac{1}{2} \frac{\delta n}{2}[(z)+z)+z B^{\prime}(z)\right] e^{-i B(z) z} \\
-\frac{1}{2} \frac{\delta n}{n_{0}(z)}\left[B(z)+z B^{\prime}(z)\right] e^{i B(z) z} & -k(z)
\end{array}\right) \Delta z .
$$

Converting to the phase-shifted wave amplitude parameters (i.e., $f \equiv F e^{\frac{1}{2} i B(z) z}$ and $\left.r \equiv e^{-\frac{1}{2} i B(z) z}\right)$ such that $|f|=|F|$ and $|r|=|R|$, serves to eliminate the exponents from the above relations. Then, Eq. (4) can take a final form as

$$
\frac{\mathrm{d}}{\mathrm{d} z}\left[\begin{array}{l}
f \\
r
\end{array}\right]=a\left[\begin{array}{l}
f \\
r
\end{array}\right]
$$

where

$a=-i\left(\begin{array}{ll}-\frac{1}{2}\left[B(z)+z B^{\prime}(z)\right]+k(z) & \frac{1}{2} \frac{\delta n}{n_{0}(z)}\left[B(z)+z B^{\prime}(z)\right] \\ -\frac{1}{2} \frac{\delta n}{n_{0}(z)}\left[B(z)+z B^{\prime}(z)\right] & \frac{1}{2}\left[B(z)+z B^{\prime}(z)\right]-k(z)\end{array}\right)$.

For an isothermal grating in which $B^{\prime}(z)=0$, Eq. (9) reverts to the original CM model (see Orfanidis [14]). It is worth noting that the contribution of $z B^{\prime}(\bar{z})$ to the cross-diagonal terms in $a$ is negligible since, typically, $z B^{\prime}(z) \ll B(z)$. However, the $z B^{\prime}(z)$ contribution to the diagonal terms is not negligible close to the Bragg condition, where $-\frac{1}{2} B(z)+k(z) \approx 0$.

\section{Analysis}

The present analysis addresses a nonisothermal FBG, with an assumed axial temperature variation, representing a thermally chirped Bragg grating. The temperature effect is taken into account through the modulation and propagation wave numbers. The modulation wavenumber varies along the axial direction as

$$
B(z)=\frac{2 \pi}{\Lambda(z)}=\frac{2 \pi}{\Lambda_{0}[1+\alpha \Delta T(z)]},
$$

where $\Lambda_{0}$ is the grating period at the reference temperature, $\alpha$ is the thermal expansion coefficient, and $\Delta T(z)$ is the excess temperature, representing the deviation from the reference temperature. The propagation wavenumber varies along the axial direction as

$$
k(z)=\frac{2 \pi}{\lambda_{0}} n(z) \approx \frac{2 \pi}{\lambda_{0}}\left[n_{0}+n_{T}^{\prime} \Delta T(z)\right],
$$

where $n_{0}$ is the refractive index at the reference temperature and $n_{T}^{\prime}$ is its derivative with respect to temperature, i.e., $n_{T}^{\prime} \equiv \frac{\mathrm{d} n}{\mathrm{~d} T}$.

The optical behavior of such a nonisothermal polymeric Bragg grating is determined by three different methods: the CM method, the thermally modified CM method (henceforth referred to as CMT), and the exact method, which is a direct numerical calculation based on Eq. (1) [or Eq. (4)] and does not contain any limiting assumptions. To obtain correct results, the direct numerical solution must be per- formed using extremely small steps $(\Delta z)$, typically 2 orders of magnitude smaller than the wavelength. To assure convergence, in the present study $\Delta z$ for the direct numerical solution was taken equal to $5.3 \mathrm{~nm}$.

The boundary conditions set the amplitude of the forward wave at the grating outlet to unity and the amplitude of the reflective wave at the outlet to zero:

$$
\left[\begin{array}{l}
F_{N} \\
R_{N}
\end{array}\right]=\left[\begin{array}{l}
1 \\
0
\end{array}\right]
$$

Linear normalization of the solution can further be applied to tune the results to different forward wave amplitudes at inlet.

For the CM and CMT methods, numerical computations are performed in $\Delta z$ steps $(j)$ :

$$
\left[\begin{array}{c}
f_{j} \\
r_{j}
\end{array}\right]=e^{-a_{j} \Delta z}\left[\begin{array}{c}
f_{j+1} \\
r_{j+1}
\end{array}\right]
$$

For each step, matrix $a$ is approximated as a constant, i.e., $a_{j}=a(z+\Delta z / 2)$, and calculated by its definition [see Eq. (9)], where for the CM method $B$ is set equal to zero. Equation (13) is then solved using the boundary conditions:

$$
\left[\begin{array}{l}
f_{N} \\
r_{N}
\end{array}\right]=\left[\begin{array}{l}
1 \\
0
\end{array}\right]
$$

Further linear normalization of results, as noted for Eq. (12), can be applied.

The numerical calculations of CM and CMT can be performed in far fewer steps (larger $\Delta z$ ) than the "exact" method since they only need to be smaller than the relevant length scale of temperature change.

\section{Results and Discussion}

In subsequent sections, thermo-optic results are given for grated fibers made of glass and polymethyl methacrylate (PMMA) polymer, respectively, and chosen to represent an extreme variation in the thermo-optic material properties of the Bragg gratings. The relevant optical and thermal parameters of these two materials are given in Table 1 [13]. It is to be noted that the values of the thermo-optic coefficient $(\mathrm{d} n / \mathrm{d} T)$ and thermal expansion coefficient $(\alpha)$ of the PMMA polymer are much larger than those of glass.

In order to examine the effect of temperature nonuniformity on the optical behavior of a grating, the impact of different temperature profiles on the power and spectral characteristics of the backward/ reflected light wave is evaluated with the aid of 


\begin{tabular}{lccc}
\hline & & Glass & PMMA \\
\hline Refractive index (at reference temperature) & $n_{0}$ & 1.4567 & 1.4853 \\
Index modulation & $\delta n$ & $7.123 \times 10^{-5}$ & $7.244 \times 10^{-5}$ \\
Index derivative with respect to temperature & $n_{T}^{\prime}$ & $8.6 \times 10^{-6} \mathrm{~K}^{-1}$ & $-1.1 \times 10^{-4} \mathrm{~K}^{-1}$ \\
Thermal expansion coefficient & $\alpha$ & $0.55 \times 10^{-6} \mathrm{~K}^{-1}$ & $73 \times 10^{-6} \mathrm{~K}^{-1}$ \\
Grating period (at reference temperature) & $\Lambda_{0}$ & $532.00 \mathrm{~nm}$ & $530.70 \mathrm{~nm}$ \\
Bragg wavelength $\left(\lambda_{B}=2 n_{0} \Lambda_{0}\right)$ & $\lambda_{B}$ & $1549.93 \mathrm{~nm}$ & $1576.50 \mathrm{~nm}$ \\
\hline
\end{tabular}

the classic CM equations, the CMT equations, and the exact numerical calculation, respectively.

Figure 1 shows the individual and aggregated effects of the mechanisms responsible for the thermooptic behavior of a thermally chirped glass Bragg grating, subjected to a steep exponential axial temperature variation, $\Delta T=85 e^{-3.344} \frac{z}{L}$, dropping from an excess temperature of $85 \mathrm{~K}$ at the inlet to just $3 \mathrm{~K}$ at the outlet of the grating. The thermomechanical effect, due to the thermal expansion of the grating, is captured in the variation of the grating pitch wavenumber $(B)$ and its axial derivative $\left(B^{\prime}\right)$. The temperature sensitivity of the refractive index $\left(n_{T}^{\prime}\right)$ is embodied in the variation of the propagating light wavenumber $(k)$.

Because of the small thermal expansion coefficient in glass, however, it is observed that the thermomechanical effect $\left(B\right.$ and $\left.B^{\prime}\right)$ is quite small in comparison to the thermo-optic effect, and that, therefore, there are only minor differences between the predictions of the CMT and the CM methods. It is noted that the magnitude of the thermo-optic effect in glass is small, due to its small thermo-optic coefficient, $n_{T}^{\prime}$ (much smaller than that of PMMA). Nevertheless, in the absence of the thermo-optic compensation that can

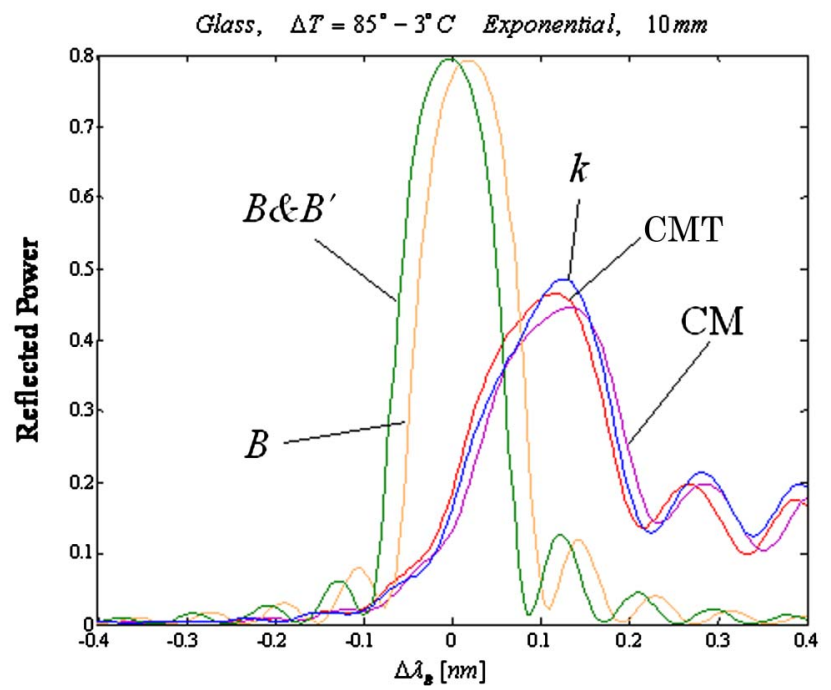

Fig. 1. (Color online) Contribution of thermo-optic effects to spectral variation (shift from Bragg wavelength) in reflected power for a $10 \mathrm{~mm}$ glass grating. A $10 \mathrm{~mm}$ glass grating under an exponential temperature profile of $\Delta T=85^{\circ}-3^{\circ} \mathrm{C}$ is considered. Calculations are separated for the sole effects of $k, B, B \& B^{\prime}$, and the combined effects models CM ( $k$ and $B$ ) and CMT ( $k$ and $\left.B \& B^{\prime}\right)$. Power is normalized to the incident light of unity. occur under small gradients in PMMA, the total Bragg shift for the glass fiber under the stated conditions reaches $0.1 \mathrm{~nm}$.

Figure 2 shows the individual and aggregated effects of the mechanisms responsible for the thermooptic behavior of a thermally chirped polymer Bragg grating, subjected to a steep exponential axial temperature variation, $\Delta T=35 e^{-3.555 \frac{z}{L}}$, dropping from an excess temperature of $35 \mathrm{~K}$ at the inlet to just $1 \mathrm{~K}$ at the outlet of the grating. It is clear from the figure that the contributions of $B$ and $k$ to the reflectivity of a PMMA grating are almost symmetrical about the Bragg wavelength, leading to significant "compensation" between these two mechanisms and only a modest net Bragg shift. However, the combined thermomechanical contribution, including the effects of both the wavenumber, $B$, and the axial derivative of the wavenumber, $B^{\prime}$, destroys this symmetry. Consequently, while the solution of the classic CM equations (without the $B^{\prime}$ contribution) suggests strong thermo-optic compensation and a very modest Bragg shift of $0.04 \mathrm{~nm}$ in this PMMA grating (see also Kim et al. [13]), inclusion of the $B^{\prime}$ term in the CMT, derived specifically to deal with large axial temperature gradients, yields a significant Bragg shift and

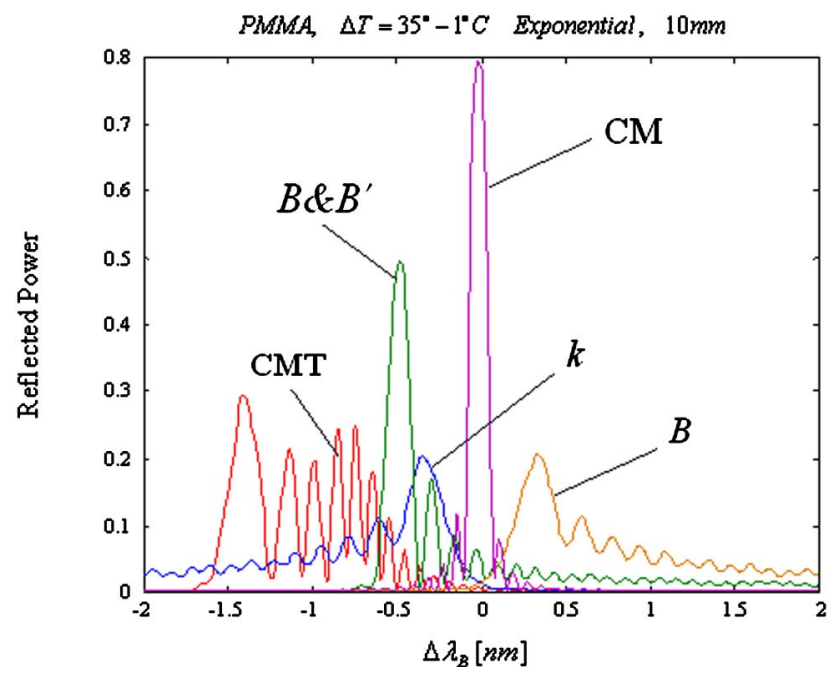

Fig. 2. (Color online) Contribution of thermo-optic effects to spectral variation (shift from Bragg wavelength) in reflected power for a 10 mm PMMA grating, under an exponential temperature profile of $\Delta T=35^{\circ}-1^{\circ} \mathrm{C}$. The individual effects of $k, B, B \& B^{\prime}$, and the combined effects models CM ( $k$ and $B$ ) and CMT ( $k$ and $\left.B \& B^{\prime}\right)$ are shown. Power is normalized to the incident light of unity. 



Fig. 3. (Color online) Normalized backward wave power for a thermally chirped $10 \mathrm{~mm}$ PMMA grating (at $\Delta \lambda_{B}=0$ and $-1.0 \mathrm{~nm}$ ) under a linear temperature profile of $\Delta T=5^{\circ}-0^{\circ} \mathrm{C}$ (start to end of grating). Calculations are according to exact numerical model, CM, and CMT. Power is normalized to the incident light of 100 units.

strong spectral "ringing" in the reflectivity of the grating.

Figure 3 presents an example of a thermally chirped Bragg grating, chosen to highlight the significant effect of temperature nonuniformity on the thermo-optic behavior of polymer grating and on the predictive inaccuracy of the classic CM model. The figure depicts the axial variation in the normalized reflected power, at the Bragg wavelength of $1576.5 \mathrm{~nm}$ [Fig. 3(a)] and at $0.1 \mathrm{~nm}$ below the Bragg wavelength [Fig. 3(b)], for a thermally chirped, $10 \mathrm{~mm}$ long, PMMA polymer FBG. The calculations were performed for an assumed linear excess temperature profile, dropping from $5 \mathrm{~K}$ above ambient at the inlet to $0 \mathrm{~K}$ at the outlet of the grating.

At the Bragg wavelength, all three techniques yield a nearly monotonic increase in reflected power as the grating inlet is approached, but while the direct numerical solution and the CMT method yield nearly identical results with a reflected power of some $9 \%$, the classic CM method substantially overpredicts the reflected power with a normalized value of 0.8. Examining Fig. 3(b), it may be seen that the close agreement between the CMT approximation and the exact solution is maintained at a $0.1 \mathrm{~nm}$ lower wavelength and reflects somewhat larger per- iodicity than seen at the Bragg wavelength. But, the results of the classic CM calculation display broad and unacceptable deviations - in the shape and magnitude of the axial power distribution. This comparison reveals that even a relatively modest axial temperature variation ( $5 \mathrm{~K}$ across a $10 \mathrm{~mm}$ grating) can produce significant "thermal chirp" effects.

The spectral variations in the power reflected by a PMMA grating, operating under different temperature profiles, i.e., a uniform temperature change of $\Delta T=35^{\circ}$, an exponential temperature profile from $10 \mathrm{~K}$ to $1 \mathrm{~K}\left(\Delta T=10 e^{-2.303} \frac{z}{L}\right)$, and a linear temperature profile decreasing from an excess temperature of $10 \mathrm{~K}$ at the inlet to zero at the outlet, are compared in Fig. 4. It is evident that in a PMMA Bragg grating, even a relatively small deviation from uniformity in temperature causes a significant shift in the reflected power and in the wavelength of the maximum reflectivity, while for a uniform temperature profile the overall thermo-optic effect is minor (small shift in reflected power versus wavelength behavior). This is due to the fact that in PMMA the effect of $k$ and $B$ result in Bragg shifts of opposite sign and nearly the same magnitude, thus leading to nearly complete compensation. However, when the axial variation in the expansion of the grating is included in the determination of the Bragg shift, through the contribution of $B^{\prime}$ to the CM solution, a decidedly asymmetric and complex spectral distribution emerges from the CMT methodology.

It is interesting to note that PMMA gratings appear to be more sensitive to a linearly varying than to an exponentially varying temperature distribution. This can be attributed to a larger average value of $z B^{\prime}(z)$ for a linear temperature profile. These pronounced effects of nonuniform temperature cannot be predicted by the original CM model since it ignores the effect of the axial gradient of the modulation wavenumber, $B^{\prime}$,

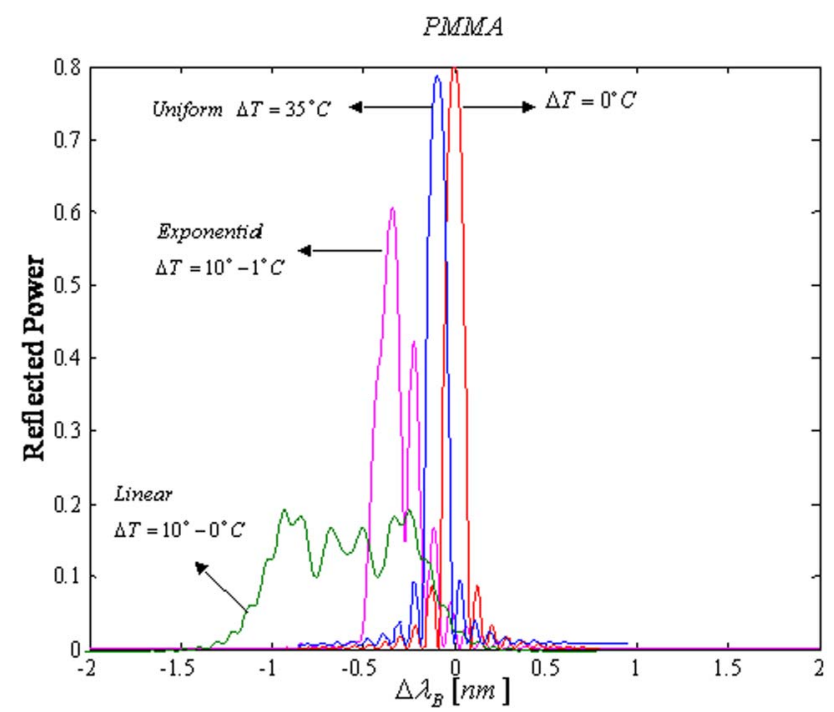

Fig. 4. (Color online) Effect of temperature nonuniformity on spectral variation (shift from Bragg wavelength) in reflected power for a $10 \mathrm{~mm}$ PMMA grating. Power is normalized to the incident light of unity. 
but are well captured by the thermally modified methodology (CMT model) presented in the previous sections.

It is also interesting to note that previous methods of accounting for chirped gratings (see the Introduction) were largely based on the original CM model. Some methods [5-7] have utilized the CM model directly, by applying it to discrete finite segments of the chirped grating. Asymptotically, as the segments become infinitely small, the method becomes, at the infinite limit, identical to the exact numerical calculation. Hence, exact results will only be obtained from calculations that are as computationally intensive as the exact numerical method. Indeed, these studies report the method to be useful only for mild chirps. These are not compatible with realistic thermal chirps in polymer fibers. Applying this method to the thermally chirped PMMA fibers would yield results largely between those obtained from the CM model to those obtained from the exact numerical method, with their numerical accuracy converging only at the computational-intensivity limit of the exact method, hence offering no practical (or other) advantage over the latter. Other methods [8,9] have suggested semianalytical approximate solutions of the CM equations for chirped gratings. These mathematical approximations were shown in those studies to be successfully close to numerical calculations of the CM equations (classical CM model) and, hence, are essentially simplifying approximations of the numerical CM model. As such, they do not offer to produce the substantially different behavior predicted by the CMT model in this study, which was verified by exact numerical calculations.

\section{Conclusions}

A thermally modified coupled-mode model (CMT) was developed to characterize the optical behavior of thermally chirped Bragg gratings. The CMT model enjoys the benefit of simplicity, as provided by the original CM model yet predicts the optical behavior of thermally chirped polymer Bragg gratings as accurately as the much more computationally intensive exact numerical calculation. Use of the CMT model has revealed the importance of accounting for the axial variation in the thermomechanical terms in the
Bragg shift calculation to capture the aperiodical distortion that can occur in nonisothermal polymer gratings.

The proposed CMT method was used to characterize the thermo-optic behavior of a PMMA FBG subjected to various nonuniform temperature profiles. It was demonstrated that even relatively small temperature nonuniformity can cause a significant shift in the reflected spectrum from the classical solution.

\section{References}

1. H. Kogelnik, "Filter response of nonuniform almost-periodic structures," Bell Syst. Tech. J. 55, 109-126 (1976).

2. C. S. Hong, J. B. Shellan, A. C. Livanos, A. Yariv, and A. Katzir, "Broad-band grating filters for thin-film optical waveguides," Appl. Phys. Lett. 31, 276-278 (1977).

3. T. Fukuzawa and M. Nakamura, "Mode coupling in thin-film chirped gratings," Opt. Lett. 4, 343-345 (1979).

4. A. C. Livanos, A. Katzir, A. Yariv, and C. S. Hong, "Chirped grating demultiplexers in dielectric waveguides," Appl. Phys. Lett. 30, 519-521 (1977).

5. M. Yamada and K. Sakuda, "Analysis of almost-periodic distributed slab waveguides via a fundamental matrix approach," Appl. Opt. 26, 3474-3478 (1987).

6. T. Erdogan, "Fiber grating spectra," J. Lightwave Technol. 15, 1277-1294 (1997).

7. L. A. Weller-Brophy and D. G. Hall, "Analysis of waveguide gratings: application of Rouard's method," J. Opt. Soc. Am. A 2, 863-871 (1985).

8. J. E. Sipe, L. Poladian, and C. M. de Sterke, "Propagation through non-uniform grating structures," J. Opt. Soc. Am. A 11, 1307-1320 (1994).

9. L. Poladian, "Graphical and WKB analysis of nonuniform Bragg gratings," Phys. Rev. E 48, 4758-4767 (1993).

10. G. D. Peng and P. L. Chu, "Polymer optical fiber photosensitivities and highly tunable fiber gratings," Fiber Integr. Opt. 19, 277-293 (2000).

11. H. Y. Liu, G. D. Peng, and P. L. Chu, "Thermal stability of gratings in PMMA and CYTOP polymer fibers," Opt. Commun. 204, 151-156 (2002).

12. I. C. M. Littler, T. Grujic, and B. J. Eggleton, "Photothermal effects in fiber Bragg gratings," Appl. Opt. 45, 4679-4685 (2006).

13. K. J. Kim, A. Bar-Cohen, and B. Han, "Thermo-optical modeling of an intrinsically heated polymer fiber Bragg grating," Appl. Opt. 46, 4357-4370 (2007).

14. S. J. Orfanidis, Electromagnetic Waves and Antennas, an online book, www.ece.rutgers.edu/ orfanidi/ewa (2004). 